Article

\title{
Removal of Cd(II) from Water by HPEI Modified Humin
}

\author{
Sanmei Li, Mingda Wu, Linghong Lu * and Jiabao Zhu \\ State Key Laboratory of Materials-Oriented Chemical Engineering, College of Chemical Engineering, \\ Nanjing Tech University, Nanjing 211816, China; sanmeilipurity@126.com (S.L.); \\ 201961104132@njtech.edu.cn (M.W.); 201861204241@njtech.edu.cn (J.Z.) \\ * Correspondence: linghonglu@njtech.edu.cn
}

Received: 13 August 2020; Accepted: 7 September 2020; Published: 25 September 2020

\begin{abstract}
Humin is the waste residue from the process of preparing humic acid, which accounts for a large proportion of the raw material (weathered coal humic acid). Its Cd(II) adsorption performance is far inferior to that of humic acid. How to regenerate humin is of great significance to the low-cost treatment of $\mathrm{Cd}$ (II) pollution in wastewater. In this study, humin was modified by hyperbranched polyethyleneimine to enhance the adsorption capacity for $\mathrm{Cd}(\mathrm{II})$. Fourier transform infrared spectroscopy and the X-ray photoelectron spectrometer showed that hyperbranched polyethyleneimine was grafted to the surface of humin. Flame atomic absorption spectroscopy showed that the saturated $\mathrm{Cd}(\mathrm{II})$ adsorption capacity of the modified humin was increased to $11.975 \mathrm{mg} / \mathrm{g}$, which is about 5 times than that of humin and is also higher than that of humic acid. The adsorption kinetics, adsorption isotherm, and thermodynamic properties of humic acid, humin, and modified humin were also studied. This study may provide a foundation for research utilizing natural resources to reduce heavy metal pollution in the environment.
\end{abstract}

Keywords: humin; hyperbranched polyethyleneimine; modification; adsorption; Cd(II)

\section{Introduction}

The development of various industries such as metallurgy, mining, and dyes has led to the increasing heavy metal contamination of the environment. Cadmium is one of the indispensable raw materials in the chemical industry; for example, it is widely used in dyes, batteries, and alloys, which leads to the entry of cadmium into the global biosphere [1,2]. The different valences of cadmium are toxic and harmful to humans and animals. Normally, cadmium flows into the human body from the circulatory system of the food chain and is then accumulated, causing serious damage to the kidneys and liver tissues. Current commonly used cadmium-containing wastewater treatments are ion exchange, membrane separation, and the adsorption method, and some involve biological treatment and the electrolysis method. Among these methods, the adsorption method is widely used due to its advantages of low cost, high efficiency [3], and easy operation [4,5].

Humic substances (HS) are of various types and often distributed in weathered coal, lignite, and peat [6,7]. HS basically have the ability to resist degradation; they can not only chemically react with heavy metal ions but also combine with clay minerals and can improve the soil and stimulate crop growth, which suggests the advantages of HS in protecting the environment. Accordingly, they are used to improve the situation of heavy metal pollution [8]. HS are usually composed of humic acid (HA), fulvic acid (FA), and humin (HM). Owning to the different solubility of components in different $\mathrm{pH}$ aqueous solutions, they can be divided into HA, which is soluble only in alkali and insoluble in acid; FA, which is soluble in both acid and alkali; and HM, which is insoluble in acid and alkali $[9,10]$. The characteristics of solubility make it easy to obtain HM and HA from HS. As a result of recent 
research, HA is widely used in the water environment [11]. Ping-I Chou et al. (2018) simulated the effect of $\mathrm{HA}$ on the release of $\mathrm{Pd}(\mathrm{II}), \mathrm{Cu}(\mathrm{II})$, and $\mathrm{Zn}$ (II) in a field and found that the presence of HA has a good inhibitory effect on the release of these heavy metal ions [12]. Although the structure of HS is still debated, it is generally believed that both HM and HA have phenolic hydroxyl and carboxyl groups. They are prone to coordination reactions with heavy metals during purification, but HA has more active groups, which improves the adsorption effect [10]. Jinjing Zhang et al. (2013) found that HA can remove heavy metals $\mathrm{Cu}$ (II) under laboratory conditions; the maximum adsorption capacities for HA were $263.2 \mathrm{mg} / \mathrm{g}$, higher than that of HM (111.1 mg/g) [13]. On the other hand, HM accounts for more than $50 \%$ of the total amount of HS, and if HM were discarded without any treatment, it would be a huge waste. Therefore, how to turn HM into a high-performance adsorption material is the key to making full use of the environmental protection and low-cost characteristics of biomass adsorption materials compared with adsorption materials such as diatomite and activated carbon.

Hyperbranched polyethyleneimine (HPEI) has a quasi-spherical structure with a large number of amino functional groups in its molecular structure, including primary, secondary, and tertiary amine groups, and is a cationic polyelectrolyte [14]. HPEI is a good adsorbent material because the amine groups on HPEI can chelate strongly with heavy metal ions $[15,16]$. However, HPEI is free in aqueous solution, and it is difficult to separate and recover when it is directly used for adsorption. Therefore, it hoped that the introduction of HPEI to modify HM will enhance the adsorption performance. Based on HPEI's characteristics of high activity and easy modification [17], it is feasible to fix HPEI on a HM matrix material with poor adsorption performance and to synthesize a more effective adsorbent material [16,17], and it is expected that the modified HM can be treated with eluent to achieve the purpose of regeneration.

The content of this paper includes (1) a comparison of the adsorption properties of HA, HM, and HM modified with HPEI and (2) Fourier transform infrared spectroscopy and X-ray photoelectron spectrometer results outlining the adsorption mechanism of the three materials.

\section{Materials and Methods}

\subsection{Materials}

Weathered coal HS (black powder, 80-100 mesh in size, $\mathrm{pH}=7.58$ ) were purchased from Beijing Aojia Fertilizer Co., Ltd. (Beijing, China). $\mathrm{HPEI}\left(\left(\mathrm{CH}_{2} \mathrm{CH}_{2} \mathrm{NH}\right)_{\mathrm{n}}, \mathrm{MW}=600,99 \%\right)$ and $\mathrm{Cd}\left(\mathrm{NO}_{3}\right)_{2}(99.9 \%)$ were both purchased from Aladdin Co., Ltd. (Seoul, Korea). Sodium hydroxide (99.6\%), hydrochloric acid $(36.5 \%)$, hydrofluoric acid $(40 \%)$, and sodium nitrate $(96 \%)$ were both provided by Sinopharm Chemical Reagent Co., Ltd. (Shanghai, China).

\subsection{Preparation of Adsorption Materials}

Alkaline acid leaching is considered to be the most commonly used and easiest method to extract HA from soil or other sediments [8]. The extraction process is simply described as follows: a certain quality of weathered coal HS were added into a conical flask, and then $0.1 \mathrm{M} \mathrm{NaOH}$ was added at a mass ratio of $m(\mathrm{HS}): \mathrm{m}(0.1 \mathrm{M} \mathrm{NaOH})=1: 10$. The conical flask was shaken on a shaker for $6 \mathrm{~h}$, then left to stand for separation. The supernatant and residue were collected separately. The residue was dissolved in $\mathrm{NaOH}$ solution, and the process was repeated 20 times to completely remove the HA and FA in the residue. Then, the residue was deashed using $10 \% \mathrm{HF}-\mathrm{HCl}$ solution. Deionized water was used to wash the residue until no $\mathrm{Cl}^{-}$was found (In order to keep the $\mathrm{pH}$ unchanged). Then, the residue was centrifuged at 6000 RPM for $20 \mathrm{~min}$, and the supernatant was poured out and dried in an oven at $35^{\circ} \mathrm{C}$ for $24 \mathrm{~h}$ to obtain HM. Next, the $\mathrm{pH}$ value of the supernatant was adjusted to 1 ; the $\mathrm{pH}$ value was measured many times by a calibrated $\mathrm{pH}$ meter (PHS-3C). $\mathrm{HCl}$ solution (6 M) was added to precipitate the solution, and then the mixture was centrifuged at $6000 \mathrm{RPM}$ for $20 \mathrm{~min}$. The insoluble $\mathrm{HM}$ was washed repeatedly with deionized water until the supernatant was neutral, and then dried in an oven at $35^{\circ} \mathrm{C}$ for $24 \mathrm{~h}$ to obtain HA. 
Modified HM was prepared using HPEI and HM at a mass ratio of 3:1, using a magnetic stirrer at $60{ }^{\circ} \mathrm{C}$ for $2,4,6$, and $8 \mathrm{~h}$ for four different samples. Samples were obtained after repeated washing and drying at $60{ }^{\circ} \mathrm{C}$ and named as 2-HM, 4-HM, 6-HM, and 8-HM. The four HPEI-modified HM, HA, and HM samples were used for the adsorption of $\mathrm{Cd}(\mathrm{II})$. A schematic illustration of the modified process and the adsorption of Cd(II) by HPEI-HM is shown in Figure 1.

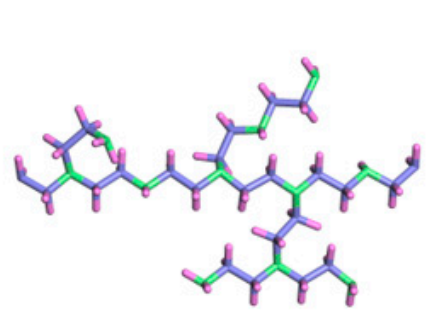

HPEI

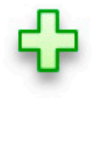

Fragment of HM

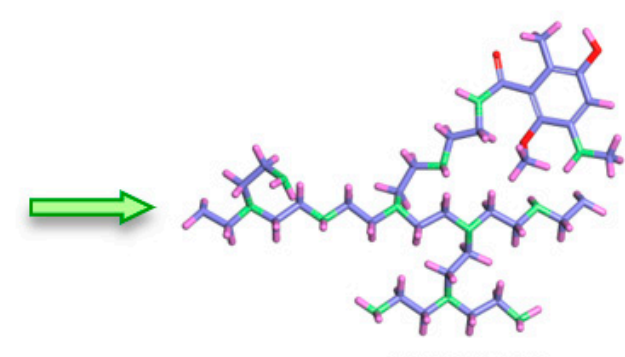

HPEI-HM
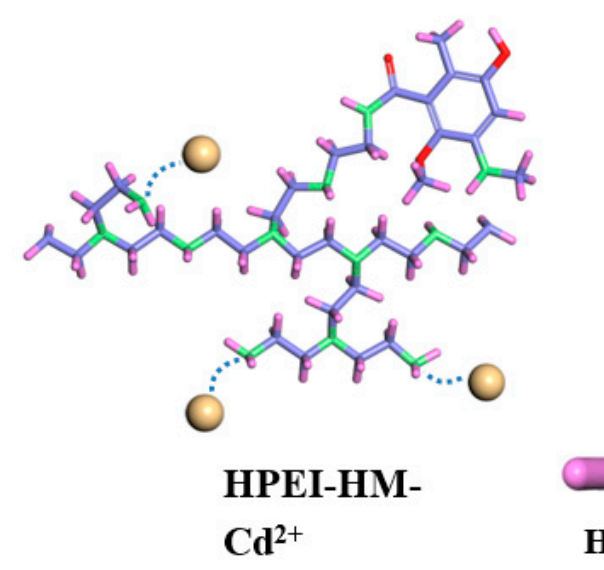

H $\quad \mathbf{N}$
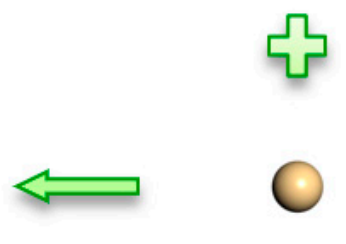

$\mathbf{C d}^{2+}$

Figure 1. Schematic illustration of synthesis processes for preparation of hyperbranched polyethyleneimine (HPEI)-humin (HM) and the adsorption of Cd(II) by HPEI-HM.

\subsection{Characterizations}

Fourier transform infrared spectroscopy (FT-IR, TENSOR 27, resolution $4 \mathrm{~cm}^{-1}$, Bruker, Billerica, MA, USA) was used to analyze the functional groups and bonding conditions on the surface of the adsorption material using $\mathrm{KBr}$ tablets. An X-ray photoelectron spectrometer (K-ALPHA, Thermo Fisher, Waltham, MA, USA) was used to analyze the surface element composition and valence state of the surface. A flame atomic absorption spectrophotometer (FLAAS) (AA900T, PerkinElmer, Waltham, MA, USA) was used to detect the concentration of Cd(II).

\subsection{Adsorption Experiments}

A total of $0.02 \mathrm{~g}$ adsorption material (HA, HM, modified HM) was added into a $50 \mathrm{~mL}$ centrifuge tube, then a $25 \mathrm{~mL} \mathrm{Cd(II)} \mathrm{solution} \mathrm{of} \mathrm{different} \mathrm{initial} \mathrm{concentration}\left(20 \mathrm{mg} / \mathrm{L}, 0.01 \mathrm{M} \mathrm{NaNO}_{3}\right.$ as background solution) for kinetic study was added. The $\mathrm{pH}$ was adjusted to 5 with $0.1 \mathrm{M} \mathrm{HCl}$ solution. The mixture was oscillated in the shaker at room temperature for different lengths of time for isotherm and thermodynamic study. For thermodynamic study, the temperature was set from 288.15 to $318.15 \mathrm{~K}$. Then the solution was obtained after 6000 RPM centrifugation and filtration using a vacuum filter bottle (with a membrane size of $0.45 \mu \mathrm{m}$ ). Finally, the Cd(II) content of the solution was determined by the FLAAS. Before that, the standard concentration of $\mathrm{Cd}(\mathrm{II})$ was measured to obtain the standard curve. 


\section{Results}

\subsection{Adsorption Performance}

It can be seen from Figure 2 that as the concentration of $\mathrm{Cd}(\mathrm{II})\left(C_{e, 0}\right)$ in the solution increases, the adsorption capacity $\left(Q_{e}\right)$ of different adsorbent materials also gradually increases. In general, the modified HM has the strongest adsorption capacity, followed by HA, while HM has the weakest. With the increase of the modification time, the adsorption capacity of modified HM for Cd(II) increases first and then decreases. At a certain concentration, the 6-HM has the highest adsorption capacity and the best adsorption effect, indicating that it is feasible to use HPEI as a modified material. Subsequently, we chose 6-HM to compare with HA and HM for further experimental research and to clarify its adsorption mechanism.

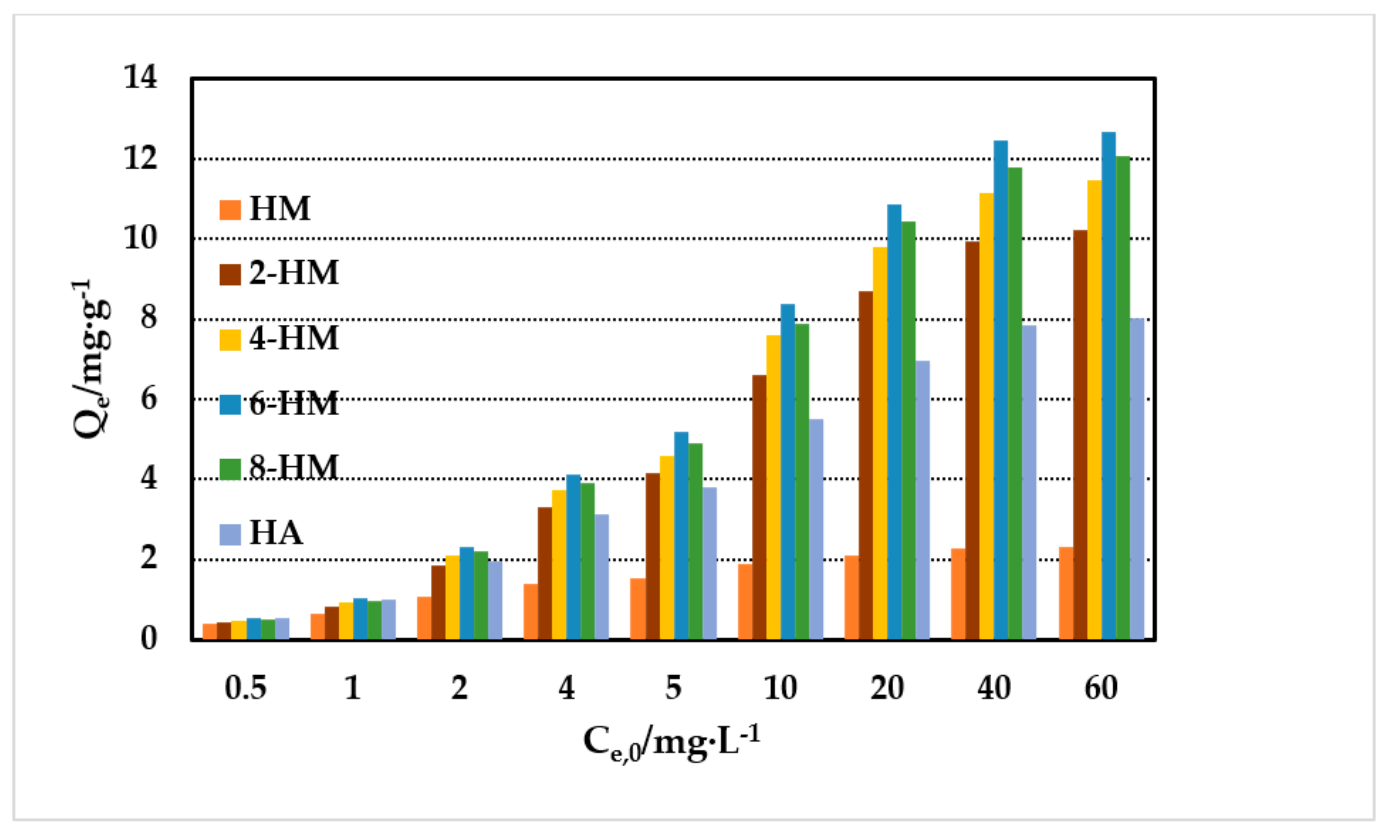

Figure 2. Comparison of adsorption capacity of different adsorption materials.

\subsection{Adsorbent Characterization}

FT-IR characterization is one of the means to obtain molecular chemical bonds or functional groups. Figure 3 shows that the surface functional groups of the three adsorbents and HPEI are abundant, and the FT-IR spectra of these three adsorbent materials are very close. The biggest difference between HM and HA is that the peak intensity at $1100-1022 \mathrm{~cm}^{-1}$ is significantly enhanced, which is attributed to the stretching vibration peak of the ether group [18], and the peak at $783 \mathrm{~cm}^{-1}$ originates from the out-of-plane bending vibration of $\mathrm{C}-\mathrm{H}$ [19]. The changes in the intensity of these peaks are also in line with the difference between the corresponding active groups of HM and HA, which also reflects the lowest adsorption performance of HM in Figure 2. After modification, it can be seen that the $\mathrm{C}=\mathrm{O}$ peak at $1702 \mathrm{~cm}^{-1}$ disappears, and a new peak is generated at $1460 \mathrm{~cm}^{-1}$. This peak also exists in the HPEI curve, which is derived from the in-plane bending vibration of C-H in the HPEI structure [20]; this indicates that the disappearance of $\mathrm{C}=\mathrm{O}$ is because the amine groups from the HPEI are reacted with the $\mathrm{C}=\mathrm{O}$ of $\mathrm{HM}$, and HPEI is successfully grafted on the surface of HM.

The surface element composition of HM before and after modification was characterized using XPS analysis. The adsorption mechanism of HM and 6-HM for Cd(II) was explored as well. As shown in Figure 4, the C1s spectrum of $\mathrm{HM}$ is composed of four fitting peaks, namely C=O,C-O, C=C, and C-C. The combined energies are 289.17, 286.05, 285.13, and $284.03 \mathrm{eV}$, respectively. For HM adsorbed with $\mathrm{Cd}(\mathrm{II})$, the $\mathrm{C} 1 \mathrm{~s}$ spectrum is also composed of four fitting peaks, $\mathrm{C}-\mathrm{O}, \mathrm{C}=\mathrm{O}, \mathrm{C}=\mathrm{C}$, and $\mathrm{C}-\mathrm{C}$. The combined 
energy centers are 289.21, 286.15, 285.45, and $284.04 \mathrm{eV}$, respectively. After adsorbing Cd(II), the content of $\mathrm{C}=\mathrm{O}$ decreased from $19 \%$ to $12.5 \%$, indicating that chemical adsorption existed and some carboxyl groups on the HM surface participated in the adsorption process.

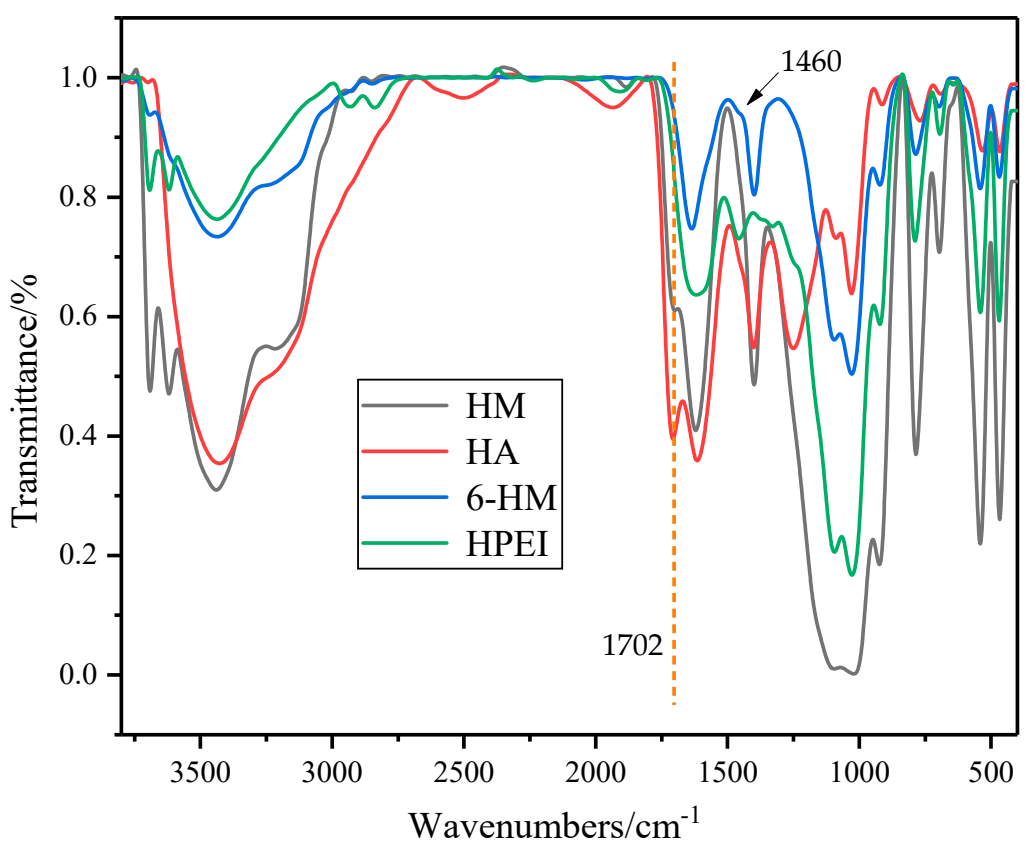

Figure 3. FT-IR of humic acid (HA), HM, and 6-HM.
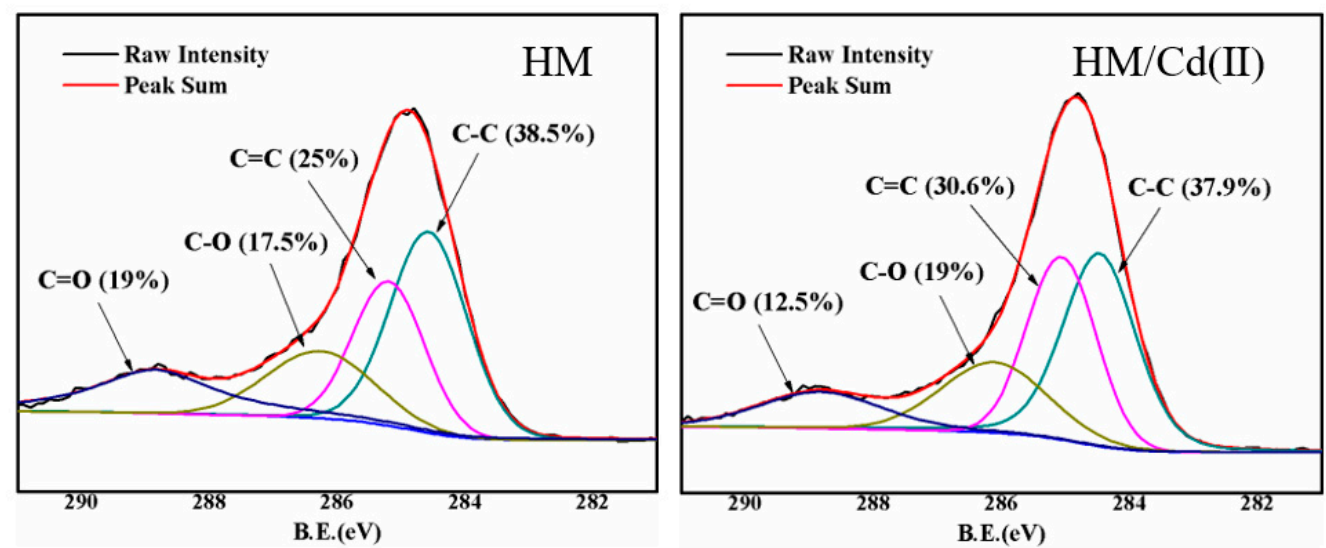

Figure 4. XPS-C1s spectra of HM adsorbent before and after Cd(II) ion adsorption.

The C1s spectra of 6-HM before and after adsorption are shown in Figure 5. Compared with HM, the peak of $\mathrm{C}=\mathrm{O}$ disappears after HPEI modification. This change is mainly owing to the reaction of the carboxyl group on the surface of HM with the amine group on HPEI.

The N1s spectra of 6-HM before and after adsorbing $\mathrm{Cd}(\mathrm{II})$ ions are shown in Figure 6. The N1s spectra is composed of three fitting peaks, specifically $\mathrm{N}-\mathrm{O}, \mathrm{N}-\mathrm{C}$, and $\mathrm{N}-\mathrm{H}$, the binding energies of which are centered at 404.21, 400.01, and $398.02 \mathrm{eV}$, respectively. By comparing the changes of the binding energy of each element peak of 6-HM before and after adsorbing $\mathrm{Cd}$ (II), it can be seen that the $\mathrm{N}-\mathrm{C}$ peak and the N-H peak are all offset. The newly added N-O bond indicates that the $\mathrm{N}$ element is involved in the adsorption process. After adsorption of $\mathrm{Cd}(\mathrm{II})$, the binding energy is increased. This shows that $\mathrm{N}$ has an obvious tendency for electron donors or shared electron pairs. The $\mathrm{N}$ atom has five electrons in its outer shell, three of which are combined to form a bond; the remaining lone pair 
is less likely to be lost but is more likely to provide the lone pair to form a complex. In the adsorption process, the $\mathrm{N}$ atom shares electrons with $\mathrm{Cd}$, leading to a decrease in electron density and an increase in binding energy.
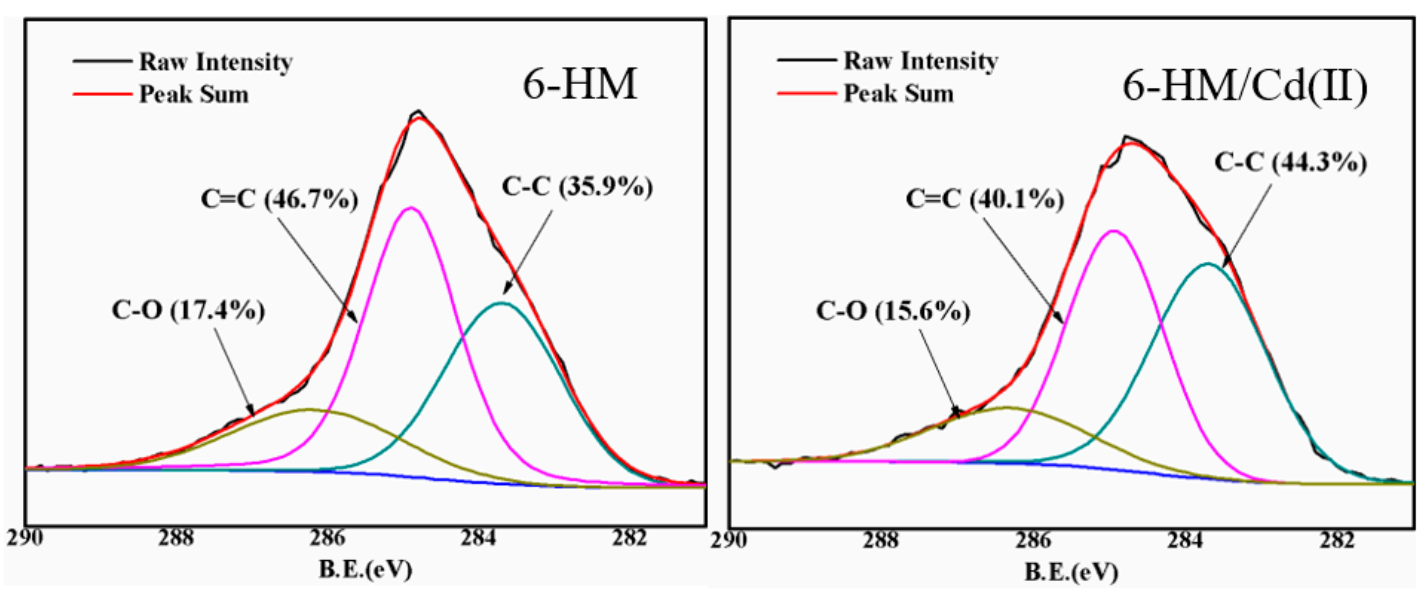

Figure 5. XPS-C1s spectra of 6-HM adsorbent before and after $\mathrm{Cd}(\mathrm{II})$ ion adsorption.

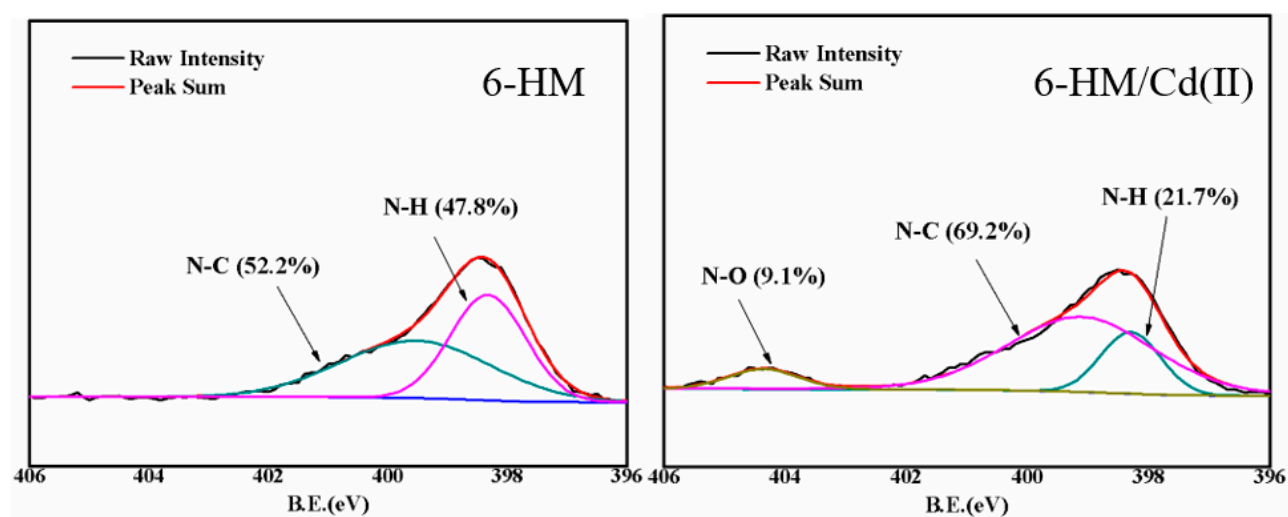

Figure 6. XPS-N1s spectra of 6-HM adsorbent before and after Cd(II) ion adsorption.

\subsection{Adsorption Kinetics}

By investigating the contact time of $\mathrm{HA}, \mathrm{HM}$, and 6-HM with $\mathrm{Cd}(\mathrm{II})$, the influence on the adsorption capacity of $\mathrm{Cd}(\mathrm{II})$ can be seen, as shown in Figure 7. The adsorption kinetics curves for the $\mathrm{Cd}(\mathrm{II})$ on the different adsorbents are roughly the same. The first $60 \mathrm{~min}$ involves fast adsorption, and $150 \mathrm{~min}$ later the adsorption equilibrium is reached. This is because in the early stage of adsorption, there are many adsorption sites on the surface of the adsorption material, and adsorption occurs rapidly. When the adsorption reaches a certain amount, the surface adsorption sites of the adsorbent decreased, which results in the decrease of the adsorption rate. During this time, the adsorption capacity and adsorption rate of 6-HM are the highest, while those of HM are the lowest. This indicates that the adsorption capacity of $\mathrm{HM}$ to $\mathrm{Cd}(\mathrm{II})$ is greatly enhanced by HPEI modification.

The adsorption results were fitted using pseudo-first-order and pseudo-second-order kinetic equations. The kinetic parameters are listed in Table 1. It can be seen that the correlation coefficients of the three pseudo-second-order kinetic equations are both close to 1 , indicating that the adsorptions of $\mathrm{HA}, \mathrm{HM}$, and 6-HM conform to the quasi-second-order kinetic equation and are mainly dominated by chemical adsorption [21]. 


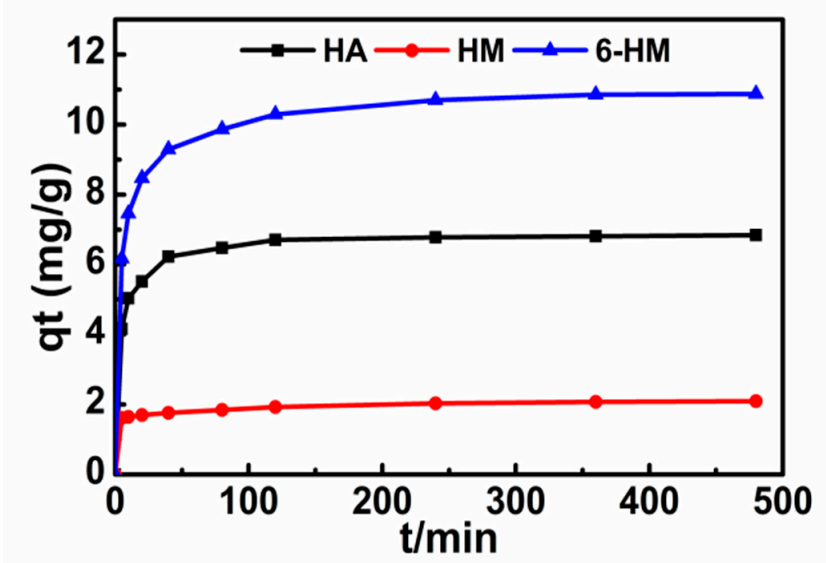

Figure 7. The $\mathrm{Cd}(\mathrm{II})$ adsorption of HA, HM, and 6-HM at different contact times.

Table 1. The parameters of adsorption kinetic model obtained by fitting the adsorption results of HA, $\mathrm{HM}$, and 6-HM.

\begin{tabular}{|c|c|c|c|c|c|c|c|}
\hline \multirow{3}{*}{ Samples } & \multirow{3}{*}{$q_{e, \exp } /(\mathrm{mg} / \mathrm{g})$} & \multirow{2}{*}{\multicolumn{3}{|c|}{$\begin{array}{l}\text { Pseudo-First-Order Kinetic } \\
\quad \operatorname{In}\left(q_{e}-q_{t}\right)=\operatorname{In} q_{e}-K_{1} t\end{array}$}} & \multicolumn{3}{|c|}{ Pseudo-Second-Order Kinetic } \\
\hline & & & & & $\frac{t}{q_{t}}=\left(\frac{1}{K_{2} q e^{2}}\right)$ & \multicolumn{2}{|c|}{$+\left(1 / q_{e}\right) t$} \\
\hline & & $q_{e, 1} /(\mathrm{mg} / \mathrm{g})$ & $k_{1} /\left(\min ^{-1}\right)$ & $\mathbf{R}^{2}$ & $q_{e, 2} /(\mathrm{mg} / \mathrm{g})$ & $k_{2} /\left(\mathrm{g} \cdot \mathrm{mg}^{-1} \cdot \min ^{-1}\right)$ & $\mathbf{R}^{2}$ \\
\hline HA & 6.838 & 1.674 & 0.016 & 0.877 & 6.897 & 0.035 & 0.999 \\
\hline HM & 2.101 & 0.489 & 0.006 & 0.989 & 2.114 & 0.071 & 0.999 \\
\hline 6-HM & 10.875 & 3.421 & 0.013 & 0.950 & 11.013 & 0.014 & 0.999 \\
\hline
\end{tabular}

\subsection{Adsorption Isotherm}

The adsorption capacity of three materials to Cd(II) was fitted by the Langmuir isotherm adsorption model and Freundlich isotherm adsorption model. The results are shown in Figure 8, and the relevant parameters of the fitting are shown in Table 2. It can be seen from Figure 8 that the equilibrium adsorption amount of the three materials increases with the increase of $\mathrm{Cd}(\mathrm{II})$ concentration $\left(C_{e}\right)$. This is because the high concentration of $\mathrm{Cd}(\mathrm{II})$ provides a greater mass transfer driving force, making it easier for $\mathrm{Cd}(\mathrm{II})$ to enter the interior of the adsorption material. There are more adsorption sites inside the adsorbent, which leads to the increase of the adsorption capacity.

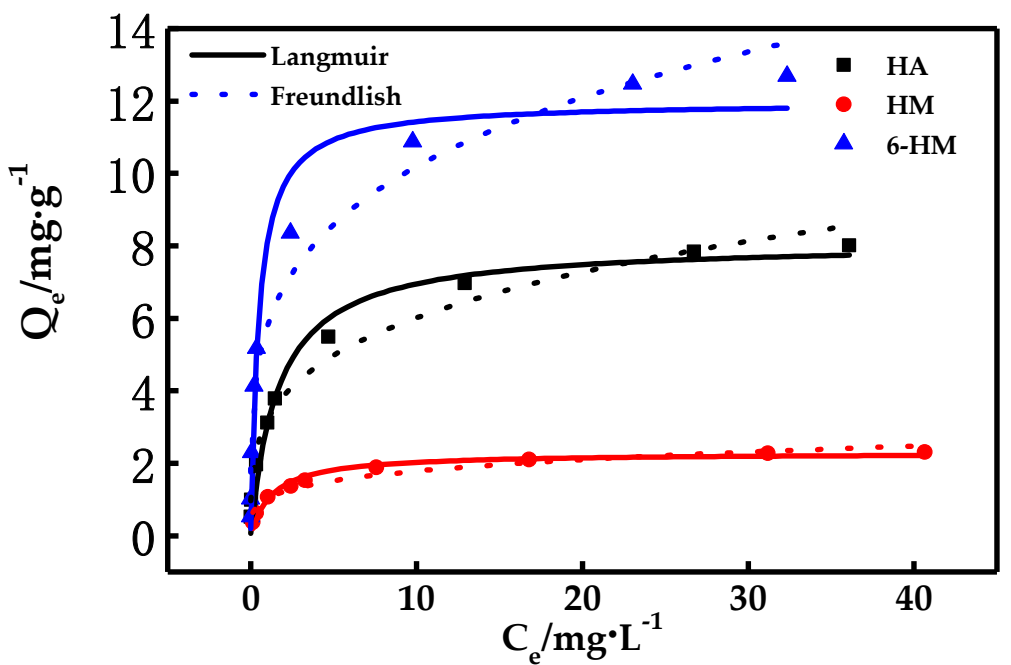

Figure 8. Adsorption isotherms of HA, HM, 6-HM and the fitting curves by the Langmuir and Freundlich isothermal adsorption models. 
Table 2. Adsorption isotherm constants of HA, HM, and 6-HM.

\begin{tabular}{|c|c|c|c|c|c|c|}
\hline \multirow[t]{2}{*}{ Samples } & \multicolumn{3}{|c|}{$\begin{array}{l}\text { Langmuir Equation } \\
\qquad Q_{e}=\frac{Q_{m} * K_{L} * C_{e}}{1+K_{L} * C_{e}}\end{array}$} & \multicolumn{3}{|c|}{$\begin{array}{l}\text { Freundlich Equation } \\
\qquad Q_{e}=K_{F} * C_{e}^{\frac{1}{n_{F}}}\end{array}$} \\
\hline & $Q_{m}(\mathrm{mg} / \mathrm{g})$ & $K_{L}(\mathrm{~L} / \mathrm{mg})$ & $\mathbf{R}^{2}$ & $K_{F}(\mathrm{~L} / \mathrm{mg})^{1 / \mathrm{n}}$ & $\frac{1}{n_{F}}$ & $\mathbf{R}^{2}$ \\
\hline HA & 8.095 & 0.480 & 0.967 & 3.284 & 0.374 & 0.9471 \\
\hline $\mathrm{HM}$ & 2.290 & 0.054 & 0.944 & 0.4383 & 0.665 & 0.9735 \\
\hline 6-HM & 11.975 & 0.380 & 0.9804 & 4.380 & 0.474 & 0.9938 \\
\hline
\end{tabular}

$Q_{m}$ : the maximum adsorption capacity of adsorbent; $K_{L}$ : the adsorption rate constant of the Langmuir isotherm adsorption equation, $\mathrm{L} / \mathrm{mg} ; K_{F}$ : the adsorption isotherm equation constant; $n_{F}$ : the adsorption strength index.

According to the fitting parameters of adsorption isotherms in Table 2, the adsorption process of $\mathrm{Cd}(\mathrm{II})$ by HM and 6-HM conforms to the Freundlich model. This indicates that the adsorption of Cd(II) by the active sites on the surface is heterogeneous and multi-layered through chemical adsorption and physical adsorption caused by Coulomb synergy [22]. The saturated adsorption amounts of 6-HM and HM in this experiment were 11.975 and $2.290 \mathrm{mg} / \mathrm{g}$, respectively. The Langmuir theoretical model of HA adsorption of Cd(II) could better fit the experimental data. According to the Langmuir model, it is suggested that the adsorption characteristic of HA to Cd(II) is single-molecule adsorption [23], and the saturated adsorption amount is $8.090 \mathrm{mg} / \mathrm{g}$. The saturated adsorption capacity of 6-HM is about 5 times than that of $\mathrm{HM}$ and is also higher than that of HA. This indicates that the modification of HM with HPEI to enhance the adsorption performance to Cd(II) was successful.

\subsection{Thermodynamic Properties}

Temperature is an important indicator to reflect the reaction efficiency [24]. To explore the influence of temperature on the adsorption of $\mathrm{HA}, \mathrm{HM}$, and 6-HM to $\mathrm{Cd}(\mathrm{II})$, the adsorption experiment was performed at 288.15, 298.15, 308.15, and $318.15 \mathrm{~K}$. The magnitude of Gibbs free energy changes $(\Delta G)$ reflects whether the chemical reaction can proceed spontaneously and can be calculated by the following two Equations (1) and (2):

$$
\begin{gathered}
\Delta G=-R T \times \ln \left(K_{d}\right) \\
\Delta G=\Delta H-T \Delta S \\
\ln \left(k_{d}\right)=\frac{\Delta S}{R}-\frac{\Delta H}{R T}
\end{gathered}
$$

where $\Delta H$ refers to the energy absorbed or released during the adsorption process. When $\Delta H>0$, the adsorption process is endothermic; when $\Delta H<0$, the adsorption process is exothermic. $\Delta S$ is the change in entropy before and after the reaction. Equation (3) can be obtained by transforming Equations (1) and (2) and then into solving the solid-liquid partition coefficient $k_{d}$ at different temperatures, and different thermodynamic parameters can be solved. To solve $k_{d}, Q_{e}$ can be fitted as the abscissa and $\ln \left(Q_{e} / C_{e}\right)$ as the ordinate for fitting. The intercept of the fitted curve is $k_{d}$, and the corresponding fitting curve is shown in Figure 9.

The thermodynamic properties obtained by fitting the experimental data are shown in Table 3. From Table 3, it can be seen that the enthalpy changes of all three adsorption materials are greater than zero. This indicates that the adsorption to $\mathrm{Cd}(\mathrm{II})$ is endothermic, so increasing temperature is beneficial to the adsorption reaction. When the temperature rises, the diffusion rate of $\mathrm{Cd}$ (II) on the surface of adsorbent becomes fast, which enhances the movement of ions. The fitting results of adsorption $\Delta S$ are greater than zero. This shows that the adsorption of $\mathrm{Cd}(\mathrm{II})$ on the three materials is a process of entropy increase. $\Delta G$ is less than 0 at the temperatures of $288.15,298.15,308.15$, and $318.15 \mathrm{~K}$, indicating that the reactions are spontaneous adsorption processes. The greater the absolute value of $\Delta G$, the more likely the adsorption reaction is to occur. This result once again shows that the higher the temperature, the easier it is for the adsorption to occur. $\Delta G$ changes with the change of temperature because the 
activity of functional groups on the surface of the adsorbent is different at different temperatures, and temperature affects the boundary layer thickness around the adsorbent.
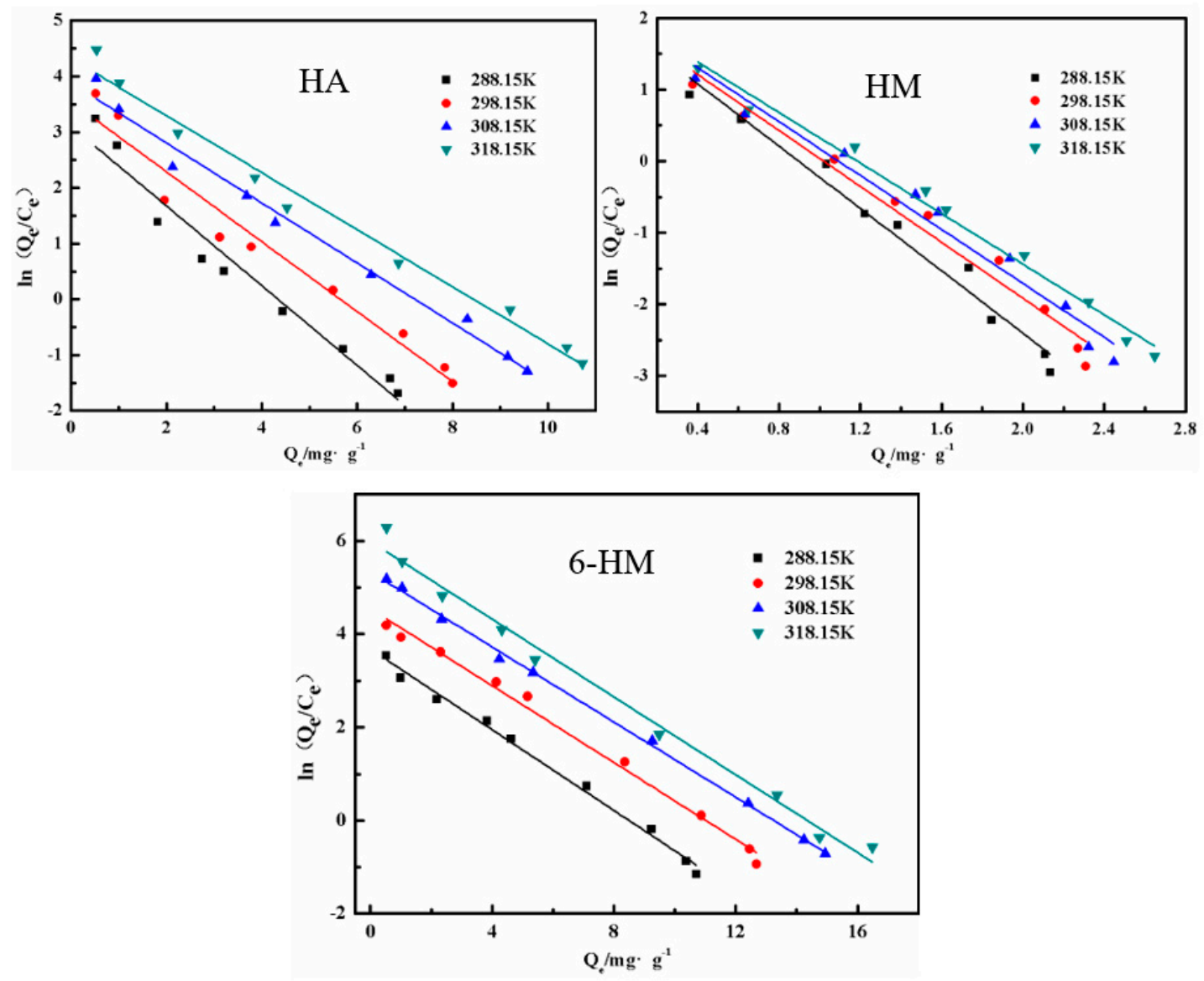

Figure 9. Thermodynamic fitting curves of HA, HM, and 6-HM.

Table 3. Thermodynamic parameters of the adsorption of HA, HM, and 6-HM to Cd(II).

\begin{tabular}{|c|c|c|c|c|c|c|}
\hline Samples & $T /(\mathrm{K})$ & $K_{d}$ & $\mathbf{R}^{2}$ & $\Delta G / \mathbf{k J} \cdot \mathrm{mol}^{-1}$ & $\Delta S / \mathrm{J} \cdot \mathrm{mol}^{-1}$ & $\Delta H / \mathrm{kJ} \cdot \mathrm{mol}^{-1}$ \\
\hline \multirow{4}{*}{ HA } & 288.15 & 3.109 & 0.956 & -2.727 & \multirow{4}{*}{37.99} & \multirow{4}{*}{8.221} \\
\hline & 298.15 & 3.546 & 0.958 & -3.107 & & \\
\hline & 308.15 & 3.883 & 0.986 & -3.487 & & \\
\hline & 318.15 & 4.32 & 0.984 & -3.867 & & \\
\hline \multirow{4}{*}{$\mathrm{HM}$} & 288.15 & 1.952 & 0.974 & -1.595 & \multirow{4}{*}{11.97} & \multirow{4}{*}{1.855} \\
\hline & 298.15 & 1.993 & 0.972 & -1.715 & & \\
\hline & 308.15 & 2.059 & 0.971 & -1.835 & & \\
\hline & 318.15 & 2.094 & 0.986 & -1.954 & & \\
\hline \multirow{4}{*}{ 6-HM } & 288.15 & 3.675 & 0.992 & -3.156 & \multirow{4}{*}{54.12} & \multirow{4}{*}{12.44} \\
\hline & 298.15 & 4.544 & 0.992 & -3.698 & & \\
\hline & 308.15 & 5.336 & 0.999 & -4.239 & & \\
\hline & 318.15 & 5.991 & 0.988 & -4.78 & & \\
\hline
\end{tabular}

\section{Conclusions}

HM and HA were obtained from HS by the alkaline acid leaching method. HM was modified using HPEI. The results of FT-IR and XPS show that HPEI was successfully grafted to the surface of HM. The XPS-N1s spectra of 6-HM adsorption before and after Cd(II) ion adsorption show that the adsorption of $\mathrm{Cd}$ (II) on modified HM is mainly owning to the strong binding energy of the $\mathrm{N}$ atom of 
N-O bond to Cd(II). The experimental results show that the saturated adsorption capacity of 6-HM reaches $11.975 \mathrm{mg} / \mathrm{g}$, which is about 5 times of the saturated adsorption amount of HM and is higher than the saturated adsorption capacity of HA. This indicates that the adsorption capacity of HM to $\mathrm{Cd}(\mathrm{II})$ is greatly enhanced by HPEI modification. The results of kinetic equation fitting show that the quasi-second-order kinetic model is more suitable for the adsorption of $\mathrm{Cd}(\mathrm{II})$ in the three materials. The adsorption of HM and 6-HM to Cd(II) is consistent with the Langmuir model, and the adsorption of $\mathrm{Cd}(\mathrm{II})$ in HA is in accordance with the Freundlich model. The results of thermodynamic properties show that the adsorption of the three adsorbents to $\mathrm{Cd}(\mathrm{II})$ is endothermic. This adsorption reaction was a spontaneous adsorption process, and increasing the temperature is helpful to the adsorption. Overall, this study may help to significantly improve the utilization of natural resources and reduce heavy metal pollution in the environment.

Author Contributions: Conceptualization, S.L. and L.L.; methodology, S.L.; software, M.W.; validation, S.L., M.W. and J.Z.; formal analysis, S.L.; investigation, S.L.; resources, L.L.; data curation, S.L. and M.W.; writing-original draft preparation, S.L. and M.W.; writing-review and editing, L.L.; visualization, S.L.; supervision, L.L.; project administration, L.L.; funding acquisition, L.L. All authors have read and agreed to the published version of the manuscript.

Funding: This research received no external funding.

Acknowledgments: This work was supported by the National Natural Science Foundation of China under Grant 21676137 and the Project of Priority Academic Program Development of Jiangsu Higher Education Institutions (PAPD).

Conflicts of Interest: The authors declare no conflict of interest.

\section{References}

1. Liu, K.; Li, F.; Cui, J.; Yang, S.; Fang, L. Simultaneous removal of Cd(II) and As(III) by graphene-like biochar-supported zero-valent iron from irrigation waters under aerobic conditions: Synergistic effects and mechanisms. J. Hazard. Mater. 2020, 395, 122623. [CrossRef] [PubMed]

2. Li, S.; Wang, M.; Zhao, Z.; Ma, C.; Chen, S. Adsorption and desorption of Cd by soil amendment: Mechanisms and environmental implications in field-soil remediation. Sustainability 2018, 10, 2337. [CrossRef]

3. Shan, C.; Ma, Z.; Tong, M. Efficient removal of free and nitrilotriacetic acid complexed Cd(II) from water by poly(1-vinylimidazole)-grafted Fe3O4@SiO2 magnetic nanoparticles. J. Hazard. Mater. 2015, 299, 479-485. [CrossRef] [PubMed]

4. Huang, Z.; Huang, Z.; Feng, L.; Luo, X.; Wu, P.; Cui, L.; Mao, X. Modified cellulose by polyethyleneimine and ethylenediamine with induced $\mathrm{Cu}(\mathrm{II})$ and $\mathrm{Pb}$ (II) adsorption potentialities. Carbohydr. Polym. 2018, 202, 470-478. [CrossRef] [PubMed]

5. Ding, L.; Lv, W.; Yao, K.; Li, L.; Wang, M.; Liu, G. Remediation of Cd(II)-contaminated soil via humin-enhanced electrokinetic technology. Environ. Sci. Pollut. Res. Int. 2017, 24, 3430-3436. [CrossRef]

6. Schwieger, A.-C.; Gebauer, K.; Ohle, A.; Beckmann, M. Determination of mercury binding forms in humic substances of lignite. Fuel 2020, 274, 117800. [CrossRef]

7. Wang, L.; Li, X.; Tsang, D.C.W.; Jin, F.; Hou, D. Green remediation of Cd and Hg contaminated soil using humic acid modified montmorillonite: Immobilization performance under accelerated ageing conditions. J. Hazard. Mater. 2020, 387, 122005. [CrossRef]

8. Javier, P.E.; Consuelo, E.; Inés, S.; Alberto, M.; Ana, M. Effects of pH conditions and application rates of commercial humic substances on $\mathrm{Cu}$ and $\mathrm{Zn}$ mobility in anthropogenic mine soils. Sustainability 2019, 11,4844 .

9. Xu, J.; Dai, Y.; Shi, Y.; Zhao, S.; Tian, H.; Zhu, K.; Jia, H. Mechanism of $\mathrm{Cr}(\mathrm{VI})$ reduction by humin: Role of environmentally persistent free radicals and reactive oxygen species. Sci. Total Environ. 2020, 725, 138413. [CrossRef]

10. Han, D.; Jiang, L.; Zhong, M.; Zhang, R.; Fu, Q.; Zhang, D.; Xia, T. Desorption and bioaccessibility of high-molecular-weight PAHs in aged field soil and humin-like fraction from a coke plant. Environ. Earth Sci. 2020, 79, 1-11. [CrossRef] 
11. Varghese, A.G.; Paul, S.A.; Latha, M.S. Remediation of heavy metals and dyes from wastewater using cellulose-based adsorbents. Environ. Chem. Lett. 2018, 17, 867-877. [CrossRef]

12. Chou, P.; Ng, D.; Li, I.; Lin, Y. Effects of dissolved oxygen, $\mathrm{pH}$, salinity and humic acid on the release of metal ions from $\mathrm{PbS}, \mathrm{CuS}$ and $\mathrm{ZnS}$ during a simulated storm event. Sci. Total Environ. 2018, 624, 1401-1410. [CrossRef] [PubMed]

13. Klučáková, M. Adsorption of nitrate on humic acids studied by flow-through coulometry. Environ. Chem. Lett. 2009, 8, 145-148. [CrossRef]

14. Bonolo, E.; Nonhlangabezo, M.; Soraya, P. Thin film composite membranes consisting of hyperbranched polyethylenimine (HPEI)-cysteamine layer for cadmium removal in water. J. Water Process Eng. 2019, 30, 100686 .

15. Zhang, J.; Wang, S.; Wang, Q.; Wang, N.; Li, C.; Wang, L. First determination of Cu adsorption on soil humin. Environ. Chem. Lett. 2012, 11, 41-46. [CrossRef]

16. Tofighy, M.A.; Mohammadi, T. Divalent heavy metal ions removal from contaminated water using positively charged membrane prepared from a new carbon nanomaterial and HPEI. Chem. Eng. J. 2020, 388, 124192. [CrossRef]

17. Liu, Y.; Xu, L.; Liu, J.; Liu, X.; Chen, C.; Li, G.; Meng, Y. Graphene oxides cross-linked with hyperbranched polyethylenimines: Preparation, characterization and their potential as recyclable and highly efficient adsorption materials for lead(II) ions. Chem. Eng. J. 2016, 285, 698-708. [CrossRef]

18. Zhou, X.; Wang, L.; Liu, X.; Xu, M.; Liu, X. Organic/inorganic hybrid consisting of supportive poly(arylene ether nitrile) microspheres and photocatalytic titanium dioxide nanoparticles for the adsorption and photocatalysis of methylene blue. Compos. Part B 2019, 177, 107414. [CrossRef]

19. Wang, B.; Yang, K.; Cheng, H.; Ye, T.; Wang, C. A hydrophobic conductive strip with outstanding one-dimensional stretchability for wearable heater and strain sensor. Chem. Eng. J. 2021, 404, 126393. [CrossRef]

20. Hua, D.; Chung, T. Universal surface modification by aldehydes on polymeric membranes for isopropanol dehydration via pervaporation. J. Membr. Sci. 2015, 492, 197-208. [CrossRef]

21. Qi, L.; Teng, F.; Deng, X.; Zhang, Y.; Zhong, X. Experimental study on adsorption of Hg(II) with microwave-assisted alkali-modified fly ash. Powder Technol. 2019, 351, 153-158. [CrossRef]

22. Wang, L.; Liu, R.; Hu, Y.; Sun, W. Adsorption of mixed DDA/NaOL surfactants at the air/water interface by molecular dynamics simulations. Chem. Eng. Sci. 2016, 155, 167-174. [CrossRef]

23. Zhang, Y.; Lu, X.; Song, D.; Liu, S. The adsorption of a single water molecule on low-index C3S surfaces: A DFT approach. Appl. Surf. Sci. 2019, 471, 658-663. [CrossRef]

24. Su, Q.; Yi, X.; Miao, J.; Chen, Y.; Chen, J.; Wang, J. A Comparative Study in Vanadium and Tungsten Leaching from Various Sources of SCR Catalysts with Local Difference. Sustainability 2020, 12, 1499. [CrossRef] 